\title{
The multi-layer free boundary problem for the $p$-Laplacian in convex domains
}

\author{
ANDREW ACKER ${ }^{\dagger}$ \\ Department of Mathematics and Statistics, Wichita State University, \\ Wichita, KS 67260-0033, USA \\ ANTOINE HENROT \\ Ecole des Mines and Institut Elie Cartan, UMR CNRS 7502 and INRIA, \\ BP 239, 54506 Vandoeuvre-les-Nancy Cedex, France \\ MichaEL POGHOSYAN ${ }^{\S}$ \\ Department of Mathematics, Yerevan State University, \\ Alex Manoogian 1, 375049, Yerevan, Armenia \\ AND \\ HENRIK SHAHGHOLIAN ${ }^{\mathrm{II}}$ \\ Department of Mathematics, Royal Institute of Technology, \\ 10044 Stockholm, Sweden
}

[Received 23 May 2002 and in revised form 16 October 2003]

\begin{abstract}
The main result of this paper concerns existence of classical solutions to the multi-layer Bernoulli free boundary problem with nonlinear joining conditions and the $p$-Laplacian as governing operator. The present treatment of the two-layer case involves technical refinements of the one-layer case, studied earlier by two of the authors. The existence treatment of the multi-layer case is largely based on a reduction to the two-layer case, in which uniform separation of the free boundaries plays a key role.
\end{abstract}

\section{Introduction and statement of the problem}

\subsection{The mathematical setting}

In this paper we continue the study of the free boundary problem arising in connection with potential flow with power-law nonlinearity (see [8,-11] for background). Mathematically, our starting point is an annular region, bounded by two convex surfaces in $\mathbb{R}^{N}(N \geqslant 2)$ :

$$
K=K_{m+2} \backslash K_{1} \quad \text { with } K_{1}, K_{m+2} \text { convex and } K_{1} \subset \subset K_{m+2} \text {. }
$$

\footnotetext{
†Email: acker@math.wichita.edu

${ }^{\ddagger}$ The second author thanks Göran Gustafsson Foundation for several visiting appointments to RIT in Stockholm. Email: henrot@iecn.u-nancy.fr

${ }^{\S}$ The third author thanks Swedish Royal Academy of Sciences for visiting appointment to RIT. Email: michael@ysu.am

${ }^{\mathbb{I}}$ Supported in part by Swedish Research Council. Email: henriks@math.kth.se
} 
The aim is to show that for a given positive integer $m$ and data

$$
\lambda_{i} \in(-1,1), \quad F_{i}(x, p, q): K \times \mathbb{R}_{+} \times \mathbb{R}_{+} \rightarrow \mathbb{R} \quad(i=1, \ldots, m)
$$

with $\lambda_{i}>\lambda_{i+1}$, one can find convex domains $K_{1} \subset \subset K_{2} \subset \subset \cdots \subset \subset K_{m+1} \subset \subset K_{m+2}$ such that the $p$-capacitary potential $u_{i}$ for each annular convex region $K_{i+1} \backslash \bar{K}_{i}$ satisfies a nonlinear joining Bernoulli condition (see the main theorems, 4.1 and 6.1)

$$
F_{i}\left(x,\left|\nabla u_{i}(x)\right|,\left|\nabla u_{i+1}(x)\right|\right)=0 \quad \text { on } \partial K_{i+1} \quad(i=1, \ldots, m) .
$$

The p-capacitary potential refers to the solution of the following Dirichlet problem:

$$
\begin{cases}\Delta_{p} u=0 & \text { in } K_{i+1} \backslash \bar{K}_{i} \\ u=\lambda_{i} & \text { on } \partial K_{i} \\ u=\lambda_{i+1} & \text { on } \partial K_{i+1}\end{cases}
$$

where $\Delta_{p}, 1<p<\infty$, is the $p$-Laplace operator defined by

$$
\Delta_{p} u:=\operatorname{div}\left(|\nabla u|^{p-2} \nabla u\right)
$$

\subsection{Applications}

The above described problem appears in several physical situations and can be appropriately interpreted in many industrial applications. A general way of interpreting it is to consider $u$ as the potential function of several adjacent flows in convex rings with prescribed pressure on the free streamlines.

A more interesting application, however, is related to the so-called Stefan problem, for large time. In this connection, the two-phase model describes crystallization (freezing) or melting of some physical substance. Multi-phase Stefan problem refers to materials capable of assuming any of three or more different states (solid, liquid, gaseous, in particular). We expand this in more detail for the two-phase case.

Let us consider a cylindrical container with the horizontal cut as the domain $K=K_{3} \backslash K_{1}$ (this is the two-dimensional case). The exterior wall $\partial K_{3}$ is kept at temperature $u=-1$, and the interior wall $\partial K_{1}$ at temperature $u=1$. The container is also filled with liquid, and the temperature of the liquid is assumed to be known initially.

Suppose the material (liquid) solidifies at temperature $-1<\lambda<1$. For simplicity we take $\lambda=0$. By continuity of the temperature for positive times, we know that there must be a curve $\Gamma(x, t)$ (for each time $t$ ) on which $u(x, t)=0$. Hence on the subregion $\{u>0\}$ the material is in liquid form and on the subregion $\{u<0\}$ the material is in solid form. Let us also assume that the temperature $u$ (depending on the material) also satisfies the (nonlinear) heat equation

$$
\Delta_{p} u-D_{t} u=0 \quad \text { in } K \backslash\{u=0\} .
$$

On the transition phase $\Gamma(x, t)$ the Stefan condition (Bernoulli condition), which follows from the energy conservation law, gives the dynamic equation of the moving curve

$$
\left|\nabla u_{1}\right|=g\left(x,\left|\nabla u_{2}\right|, V\right),
$$


where $u_{1}$ and $u_{2}$ represent the function $u$ on $\{u>0\}$ and on $\{u<0\}$ respectively. Here $V$ is the normal velocity of the curve $\Gamma(x, t)$, and the nonlinear joining condition (3) may depend on the density of the heat source over the inter-phase boundary (due for instance to an extra super-heating).

For large time, the heat flux tends to stabilize and becomes stationary. Hence $u_{t}$ and $V$ both become approximately zero. Therefore the realistic model for the stationary problem is the one given by

$$
\Delta_{p} u=0 \quad \text { in } K \backslash\{u=0\}, \quad\left|\nabla u_{1}\right|=g\left(x,\left|\nabla u_{2}\right|, 0\right) \quad \text { on }\{u=0\} .
$$

It is noteworthy that the $p$-Laplace operator constitutes a subclass of a larger class of operators, appearing in many modeling problems in industrial applications, due to non-Newtonian behavior of fluids.

For further applications, and background in the case $p=2$, we refer the reader to [1,2], and the references therein.

\subsection{Main result}

We prove existence and $C^{1}$ regularity of the free boundary in the two-phase case. More precisely, the main result of this paper is the following:

THEOREM 1.1 (two phases) Let $K_{1}, K_{3}$ be two convex domains such that $K_{1} \subset \subset K_{3}$, and $g$ : $\left(K_{3} \backslash K_{1}\right) \times \mathbb{R}_{+} \rightarrow \mathbb{R}_{+}$a continuous positive function, nondecreasing with respect to its second argument and satisfying some concavity property (see Definition 2.3 for a precise statement). Then there exists a convex $C^{1}$ domain $\omega, K_{1} \subset \subset \omega \subset \subset K_{3}$, which is a classical solution of the two-layer free boundary problem. The latter means that the $p$-capacitary potentials $u_{1}$ and $u_{2}$ of the sets $\omega \backslash \bar{K}_{1}$ and $K_{3} \backslash \bar{\omega}$ respectively, i.e. solutions of

$$
\left\{\begin{array} { l l } 
{ \Delta _ { p } u _ { 1 } = 0 } & { \text { in } K _ { 2 } \backslash \overline { K } _ { 1 } , } \\
{ u _ { 1 } = 1 } & { \text { on } \partial K _ { 1 } , } \\
{ u _ { 1 } = 0 } & { \text { on } \partial \omega , }
\end{array} \quad \left\{\begin{array}{ll}
\Delta_{p} u_{2}=0 & \text { in } K_{3} \backslash \bar{\omega}, \\
u_{2}=-1 & \text { on } \partial K_{3}, \\
u_{2}=0 & \text { on } \partial \omega,
\end{array}\right.\right.
$$

with $\Delta_{p}, 1<p<\infty$, the $p$-Laplace operator, satisfy

$$
\lim _{\substack{z \rightarrow x \\ z \in \omega \backslash \bar{K}_{1}}}\left|\nabla u_{1}(z)\right|=\lim _{\substack{y \rightarrow x \\ y \in K_{3} \backslash \bar{\omega}}} g\left(y,\left|\nabla u_{2}(y)\right|\right) \quad \forall x \in \partial \omega .
$$

Section 2 is devoted to describing the possible nonlinear joining conditions we are able to handle. In Section 3, we give some useful auxiliary results. Section 4 is devoted to the proof of the main theorem, Section 5 is the separation result and Section 6 describes extension to the multi-phase case.

\section{The nonlinear joining condition}

In this section, we discuss what could be the nonlinear joining condition involving $\nabla u_{i}$ and $\nabla u_{i+1}$ at the interface $\gamma_{i}=\partial K_{i+1}$ between the two phases. We recall that this condition is written in the general form

$$
F_{i}\left(x,\left|\nabla u_{i}(x)\right|,\left|\nabla u_{i+1}(x)\right|\right)=0 \quad(i=1, \ldots, m)
$$

with $F_{i}: K \times \mathbb{R}_{+} \times \mathbb{R}_{+} \rightarrow \mathbb{R}$. 
We will always assume that $F_{i}(x, p, q)$ is a continuous function on $K \times \mathbb{R}_{+} \times \mathbb{R}_{+}$, and that $F_{i}(x, p, q)$ is strictly increasing as a function of variable $p$ for all $x, q$.

This assumption and the implicit function theorem allow us to write the joining condition (5) in the following equivalent form

$$
\left|\nabla u_{i}(x)\right|=g_{i}\left(x,\left|\nabla u_{i+1}(x)\right|\right)
$$

where $g_{i}: K \times \mathbb{R}_{+} \rightarrow \mathbb{R}_{+}$are given functions.

An important tool, used in the proof of our main theorem and due to [13] and [4], is the following property: if $\gamma_{i}$ contains a line segment $I$ then $x \mapsto 1 /\left|\nabla u_{i}(x)\right|$ is a convex function while $x \mapsto$ $1 /\left|\nabla u_{i+1}(x)\right|$ is a concave function on $I$ (see Lemma 3.12). This in conjunction with concavity assumption on the function $x \mapsto 1 / g_{i}\left(x,\left|\nabla u_{i+1}(x)\right|\right)$ underlies one of the main techniques in the proof of our main result. Therefore, the property that $g_{i}$ must satisfy is the following:

$$
x \mapsto 1 / g_{i}(x, q(x)) \text { is a concave function as soon as } 1 / q(x) \text { is a concave function. }
$$

For general functions $g$ we cannot expect to have convexity of the level sets of the solution. In fact the first author (see [3]) obtained an example of the convex two-layer problem in the plane for which no convex solution exists corresponding to the joining condition in the form: $\left|\nabla u_{2}(x)\right|^{2}-$ $\left|\nabla u_{1}(x)\right|^{2}=\lambda^{2}$ (cf. $(8)$ ). Laurence and Stredulinsky [13] gave an example of the convex two-layer problem with the same joining condition such that the natural variational minimizer is not convex.

It is also an open question whether there exist classical solutions at all, for general (regular) functions $g$. In two space dimensions this was settled by H. W. Alt and L. A. Caffarelli [6]. So the question to be raised is what are the "necessary and sufficient" conditions to have existence of convex classical solutions. Our condition (7) may seem somewhat artificial, but it is the only working condition at this moment. Let us remark that a similar "convexity condition" was assumed in [2] and [5].

EXAMPLE The classical nonlinear joining condition (see e.g. [1], [13]) is given by

$$
\left|\nabla u_{i}(x)\right|^{\alpha}-\left|\nabla u_{i+1}(x)\right|^{\alpha}=a_{i}(x)^{\alpha}
$$

where $\alpha \geqslant 1$ and $a_{i}(x)>0$. This joining condition satisfies the "convexity condition" (7) provided that the function $1 / a_{i}$ is concave, as will follow from Lemma 2.1 below. (Regarding applicability of other convexity conditions, we refer to [2, Example 2.9] and [5, Example 4.7].)

LEMMA 2.1 Let $a$ and $q$ be positive functions defined on $\mathbb{R}^{N}$ and such that $1 / a$ and $1 / q$ are concave. Then, for $\alpha \geqslant 1$, the function $x \mapsto 1 /\left(a(x)^{\alpha}+q(x)^{\alpha}\right)^{1 / \alpha}$ is concave.

Proof. It is sufficient to do the proof for $C^{1}$ functions $a$ and $q$, since the result will follow for less regular functions by a simple density argument just using pointwise convergence.

Let us set

$$
f(x):=\frac{1}{\left(a(x)^{\alpha}+q(x)^{\alpha}\right)^{1 / \alpha}},
$$

which is a $C^{1}$ function. We want to prove the following inequality:

$$
\forall x, y \in \mathbb{R}^{N} \quad(\nabla f(x), y-x) \geqslant f(y)-f(x) .
$$


Now

$$
(\nabla f(x), y-x)=-\frac{1}{\left(a(x)^{\alpha}+q(x)^{\alpha}\right)^{1+1 / \alpha}}\left[a(x)^{\alpha-1}(\nabla a(x), y-x)+q(x)^{\alpha-1}(\nabla q(x), y-x)\right] .
$$

By concavity of $1 / a$ we have

$$
\left(\nabla\left(\frac{1}{a}\right)(x), y-x\right)=-\frac{1}{a(x)^{2}}(\nabla a(x), y-x) \geqslant \frac{1}{a(y)}-\frac{1}{a(x)} .
$$

A similar inequality holds for $1 / q$. Putting these in 10 yields

$$
\begin{aligned}
& (\nabla f(x), y-x) \\
& \quad \geqslant \frac{1}{\left(a(x)^{\alpha}+q(x)^{\alpha}\right)^{1+1 / \alpha}}\left[a(x)^{\alpha+1}\left(\frac{1}{a(y)}-\frac{1}{a(x)}\right)+q(x)^{\alpha+1}\left(\frac{1}{q(y)}-\frac{1}{q(x)}\right)\right],
\end{aligned}
$$

that is,

$$
(\nabla f(x), y-x) \geqslant \frac{1}{\left(a(x)^{\alpha}+q(x)^{\alpha}\right)^{1+1 / \alpha}}\left(\frac{a(x)^{\alpha+1}}{a(y)}+\frac{q(x)^{\alpha+1}}{q(y)}\right)-f(x) .
$$

So, to prove (9), it remains to prove the following inequality:

$$
\frac{1}{\left(a(x)^{\alpha}+q(x)^{\alpha}\right)^{1+1 / \alpha}}\left(\frac{a(x)^{\alpha+1}}{a(y)}+\frac{q(x)^{\alpha+1}}{q(y)}\right) \geqslant \frac{1}{\left(a(y)^{\alpha}+q(y)^{\alpha}\right)^{1 / \alpha}} .
$$

Let us set $x_{1}=a(x) / a(y), x_{2}=q(x) / q(y), t_{1}=a(x)^{\alpha}, t_{2}=q(x)^{\alpha}$. Inequality (11) can be rewritten as

$$
x_{1}^{\alpha} x_{2}^{\alpha} \frac{\frac{1}{t_{1}}+\frac{1}{t_{2}}}{\frac{x_{1}^{\alpha}}{t_{1}}+\frac{x_{2}^{\alpha}}{t_{2}}} \leqslant\left(\frac{t_{1} x_{1}+t_{2} x_{2}}{t_{1}+t_{2}}\right)^{\alpha}
$$

or

$$
\frac{t_{1}+t_{2}}{t_{1} x_{2}^{\alpha}+t_{2} x_{1}^{\alpha}} \leqslant\left(\frac{\frac{t_{1}}{x_{2}}+\frac{t_{2}}{x_{1}}}{t_{1}+t_{2}}\right)^{\alpha} .
$$

Now, the inequality between harmonic and arithmetic means yields

$$
\left(\frac{t_{1}+t_{2}}{\frac{t_{1}}{x_{2}}+\frac{t_{2}}{x_{1}}}\right)^{\alpha} \leqslant\left(\frac{t_{1} x_{2}+t_{2} x_{1}}{t_{1}+t_{2}}\right)^{\alpha}
$$

and the inequality 12 follows immediately using the convexity property of the function $x \mapsto x^{\alpha}$.

We present now another class of functions $g_{i}$ with the above-mentioned property.

LEMMA 2.2 Assume that the function $g: \mathbb{R}_{+}^{2} \rightarrow \mathbb{R}_{+}$satisfies the following set of hypotheses:

(H1) $g$ is concave,

(H2) $g$ satisfies the following inequality:

$$
\forall\left(\xi_{1}, \xi_{2}, \eta_{1}, \eta_{2}\right) \in \mathbb{R}_{+}^{4} \quad g\left(\xi_{1}, \eta_{1}\right) g\left(\xi_{2}, \eta_{2}\right) \geqslant g\left(\sqrt{\xi_{1} \xi_{2}}, \sqrt{\eta_{1} \eta_{2}}\right)^{2} .
$$


If $a: \mathbb{R}^{N} \rightarrow \mathbb{R}_{+}, q: \mathbb{R}^{N} \rightarrow \mathbb{R}_{+}$are two given functions such that $1 / a$ and $1 / q$ are concave, then $x \mapsto 1 / g(a(x), q(x))$ is concave.

Proof. Again, it suffices to prove this for $C^{1}$ functions $g, a$ and $q$. Let us set

$$
f(x):=\frac{1}{g(a(x), q(x))},
$$

which is a $C^{1}$ function. We prove the following inequality (cf. (9)):

$$
\forall x, y \in \mathbb{R}^{N} \quad(\nabla f(x), y-x) \geqslant f(y)-f(x) .
$$

Now

$$
\begin{aligned}
& (\nabla f(x), y-x) \\
& =\frac{-1}{g(a(x), q(x))^{2}}\left[\frac{\partial g}{\partial \xi}(a(x), q(x))(\nabla a(x), y-x)+\frac{\partial g}{\partial \eta}(a(x), q(x))(\nabla q(x), y-x)\right],
\end{aligned}
$$

and by the concavity assumption on $1 / a$ and $1 / q$ (see the proof of Lemma 2.1) we have

$$
\begin{aligned}
(\nabla f(x), y-x) \geqslant & \frac{1}{g(a(x), q(x))^{2}}\left[\frac{\partial g}{\partial \xi}(a(x), q(x)) a(x)^{2}\left(\frac{1}{a(y)}-\frac{1}{a(x)}\right)\right. \\
& \left.+\frac{\partial g}{\partial \eta}(a(x), q(x)) q(x)^{2}\left(\frac{1}{q(y)}-\frac{1}{q(x)}\right)\right]
\end{aligned}
$$

Next, using concavity of $g$ we arrive at

$$
\frac{\partial g}{\partial \xi}\left(\xi_{1}, \eta_{1}\right)\left(\xi_{2}-\xi_{1}\right)+\frac{\partial g}{\partial \eta}\left(\xi_{1}, \eta_{1}\right)\left(\eta_{2}-\eta_{1}\right) \geqslant g\left(\xi_{2}, \eta_{2}\right)-g\left(\xi_{1}, \eta_{1}\right)
$$

where $\left(\xi_{1}, \eta_{1}\right)=(a(x), q(x))$ and $\left(\xi_{2}, \eta_{2}\right)=\left(a(x)^{2} / a(y), q(x)^{2} / q(y)\right)$. Inequality 14 now follows immediately from this and inequality $[13$.

EXAMPLES 1 . The function $g(\xi, \eta):=\left(\xi^{\alpha}+\eta^{\alpha}\right)^{1 / \alpha}$ which corresponds to the classical nonlinear joining condition already mentioned does not fall into the above framework when $\alpha \geqslant 1$ (because $g$ is not concave), but for $0 \leqslant \alpha<1$. Indeed, in this case assumption (H1) is easily verified by proving that the Hessian of $g$ is negative on $\mathbb{R}_{+}^{2}$. As for $(\mathrm{H} 2)$, it follows immediately from the inequality

$$
\left(\xi_{1} \eta_{2}\right)^{\alpha}+\left(\xi_{2} \eta_{1}\right)^{\alpha} \geqslant 2\left(\xi_{1} \xi_{2} \eta_{1} \eta_{2}\right)^{\alpha / 2}
$$

which gives

$$
\left[\left(\xi_{1} \xi_{2}\right)^{\alpha}+\left(\eta_{1} \eta_{2}\right)^{\alpha}+\left(\xi_{1} \eta_{2}\right)^{\alpha}+\left(\xi_{2} \eta_{1}\right)^{\alpha}\right]^{1 / \alpha} \geqslant\left[\left(\xi_{1} \xi_{2}\right)^{\alpha}+\left(\eta_{1} \eta_{2}\right)^{\alpha}+2\left(\xi_{1} \xi_{2} \eta_{1} \eta_{2}\right)^{\alpha / 2}\right]^{1 / \alpha},
$$

which is inequality 13 .

2. The function $g(\xi, \eta):=\xi^{\alpha} \eta^{\beta}$ with $\alpha \geqslant 0, \beta \geqslant 0$ and $\alpha+\beta \leqslant 1$ can also be considered. Conditions (H1), (H2) are readily verified in this case.

3. More generally, we can consider a function like $g(\xi, \eta):=\sum_{i \in I} a_{i} \xi^{\alpha_{i}} \eta^{\beta_{i}}$ (finite or infinite sum) with $a_{i} \geqslant 0, \alpha_{i} \geqslant 0, \beta_{i} \geqslant 0$ and $\alpha_{i}+\beta_{i} \leqslant 1$. Assumption (H1) is elementary ( $g$ is a 
combination of concave functions with positive coefficients) while inequality $[13$ is obtained from the expansion of

$$
\sum_{i_{1} \in I} \sum_{i_{2} \in I} a_{i_{1}} a_{i_{2}}\left(\xi_{1}^{\alpha_{i_{1}} / 2} \xi_{2}^{\alpha_{i_{2}} / 2} \eta_{1}^{\beta_{i_{1}} / 2} \eta_{2}^{\beta_{i_{2}} / 2}-\xi_{1}^{\alpha_{i_{2}} / 2} \xi_{2}^{\alpha_{i_{1}} / 2} \eta_{1}^{\beta_{i_{2}} / 2} \eta_{2}^{\beta_{i_{1}} / 2}\right)^{2} \geqslant 0 .
$$

Definition 2.3 Define $\mathcal{G}$ to be the family of all functions $g: K \times \mathbb{R}_{+} \rightarrow \mathbb{R}_{+}$satisfying the following conditions:

(A1) $g$ is continuous and $\exists c_{0}>0$ such that $g(x, 0) \geqslant c_{0}$ for all $x \in K$,

(A2) $g$ is nondecreasing with respect to the second argument,

(A3) $g$ has the following concavity property: $x \mapsto 1 / g(x, q(x))$ is concave whenever $q$ is a given function such that $1 / q$ is concave, and

(A4) for any given value $y_{0}>0$, there exist constants $0<C_{1}<C_{2}$ such that $C_{1} \leqslant g(x, y) / y \leqslant$ $C_{2}$, uniformly for all $x \in K$ and all $y \geqslant y_{0}$.

Henceforward we will always consider the following nonlinear joining condition:

$$
\left|\nabla u_{i}(x)\right|=g_{i}\left(x,\left|\nabla u_{i+1}(x)\right|\right),
$$

with $g_{i} \in \mathcal{G}$.

\section{Preliminary results}

In this section we will sum up some of the auxiliary results used in this paper. We remark that the usual comparison and maximum principle for elliptic partial differential equations is one of the basic tools here; see [17].

Lemma 3.1 (Exterior Barrier) Let $D$ be a convex domain in $\mathbb{R}^{N}$ and suppose $u$ is a continuous nonnegative function on $B\left(x^{0}, r\right), p$-harmonic in $B\left(x^{0}, r\right) \cap D$, with $x^{0} \in \partial D$. Let also $u=0$ on $\partial D \cap B\left(x^{0}, r\right)$. If $\partial D$ is not $C^{1}$ at $x^{0}$, i.e. $D$ has (at least) two supporting planes at $x^{0}$, then

$$
\lim _{x \rightarrow x^{0}}|\nabla u(x)|=0, \quad x \in D .
$$

Lemma 3.2 (Interior Barrier) Let $D$ be a convex domain in $\mathbb{R}^{N}$ and suppose $u$ is a continuous nonnegative function on $B\left(x^{0}, r\right), p$-harmonic in $B\left(x^{0}, r\right) \backslash D$, with $x^{0} \in \partial D$. Let also $u=0$ on $\partial D \cap B\left(x^{0}, r\right)$. If

$$
|\nabla u| \leqslant C_{0} \quad \text { in } B\left(x^{0}, r\right) \backslash D,
$$

then $\partial D \cap B\left(x^{0}, r / 2\right)$ is $C^{1}$ with a uniform $C^{1}$-norm, i.e. there exists a constant $C_{1}=C_{1}\left(C_{0}, N\right)$ such that

$$
|\nabla \psi(x)-\nabla \psi(y)| \leqslant C_{1},
$$

where $\psi$ is a map that represents $\partial D$ near $x^{0} \in \partial D$.

The proofs of these lemmas follow from standard theory using barriers at conical boundary points. The existence of such barriers is proven in [7] (see also [12]).

Remark 3.3 By Lemmas 3.1 and 3.2 if $C_{0}^{-1} \leqslant|\nabla u| \leqslant C_{0}$ in $D$, $\partial D$ must be $C^{1}$ with $C^{1}$-norm depending on $C_{0}$. 
DEFInition 3.4 (Blow-up) For the functions $u^{j}: B\left(x^{j}, 1\right) \rightarrow \mathbb{R}$ and for a sequence $\left\{r_{j}\right\}$ of nonnegative numbers $\left(r_{j} \rightarrow 0\right)$ we define the scaled functions on $B\left(0,1 / r_{j}\right)$ by

$$
\tilde{u}^{j}(x)=\frac{u^{j}\left(r_{j} x+x^{j}\right)-u^{j}\left(x^{j}\right)}{r_{j}} .
$$

Obviously, if all functions $u^{j}$ are Lipschitz-continuous in $B\left(x^{j}, 1\right)$ with the same constant, then $\tilde{u}^{j}$ are uniformly Lipschitz in $B(0, R)\left(R<1 / r_{j}\right)$. Thus, there exists a subsequence $n_{k}$ such that $\tilde{u}^{n_{k}}$ converges locally in $C^{\alpha}\left(\mathbb{R}^{N}\right)$ to a function $u^{0}$. Moreover, if $u^{j}$ are $p$-harmonic, then so is $u^{0}$ in $\left\{u^{0}>0\right\}$ and $u^{0}(0)=0$.

Lemma 3.5 Let $S\left(C_{0}\right)$ be the set of all $C^{1}$ domains $D \subset \mathbb{R}_{+} \times \mathbb{R}^{N-1}$ such that $B(0,1) \cap D$ is convex, $0 \in \partial D$ and $\|\partial D\|_{C^{1}(B(0,1 / 2))} \leqslant C_{0}$. Then any blow-up of a sequence $D_{j} \in S\left(C_{0}\right)$ converges to a half space, i.e., if $r_{j} \downarrow 0$ and $D_{j} \in S\left(C_{0}\right)$, then for $\tilde{D}_{j}:=\left(1 / r_{j}\right) D_{j}=\left\{x: r_{j} x \in D_{j}\right\}$ we have

$$
\lim \sup \tilde{D}_{j}=\overline{\mathbb{T}}
$$

where $\mathbb{T}=\left\{x_{1}>0\right\}$, and lim sup means the set of all limit points of sequences $\left\{x^{j}\right\}$ with $x^{j} \in \tilde{D}_{j}$.

LEMMA 3.6 Let $u^{j}$ be the $p$-capacitary potential of an annular domain $D_{j}=D_{j}^{2} \backslash D_{j}^{1}$ with convex uniform $C^{1}$ boundaries. Suppose moreover the gradient of $u^{j}$ satisfies

$$
\left|\nabla u^{j}(x)\right| \leqslant \Lambda_{0}<\infty
$$

uniformly both in $j$ and $x \in D_{j}$. Then any convergent subsequence of $\tilde{u}^{r_{j}}$ at any boundary point gives a linear function $u^{0}=\alpha x_{1}^{+}$, after suitable rotation and translation. In particular, for any boundary point $x^{j} \in \partial D_{j}$,

$$
u^{j}\left(y+x^{j}\right)=u^{j}\left(x^{j}\right)+\alpha y_{1}^{+}+o\left(r_{j}\right)
$$

in $B\left(0, r_{j}\right)$, in some rotated system.

The proof of this lemma is just the same as the proof of Lemma 2.4 in [9]. The uniformity in norms is crucial.

Using these lemmas, we can prove the following (cf. [11, Theorem 1.3]).

LEMMA 3.7 Let $D_{1}$ and $D_{2}$ be two nested open convex domains $\left(\bar{D}_{1} \subset D_{2}\right)$, and $u$ denote the $p$-capacitary potential of $D=D_{2} \backslash \bar{D}_{1}$. Then for $x \in \partial D$,

$$
\lim _{y \rightarrow x}|\nabla u(y)| \quad \text { exists }
$$

nontangentially (with values in $[0, \infty]$ ). In particular $|\nabla u|$ can be defined (with values in $[0, \infty]$ ) up to the boundary $\partial D$ as nontangential limit. Moreover, $|\nabla u|$ is upper semicontinuous up to $\partial D_{2}$ and lower semicontinuous up to $\partial D_{1}$.

Proof. Since the problem is local, depending on whether we are close to $\partial D_{1}$ or $\partial D_{2}$, we may start with point $x^{0} \in \partial D_{1}$. In case $|\nabla u|$ is bounded in a neighborhood of $x^{0}$ the proof was given in Theorem 1.3 of [11]. So suppose there exists a sequence $x^{j} \in D$ with

$$
\lim _{j} x^{j}=x^{0}, \quad\left|\nabla u\left(x^{j}\right)\right| \rightarrow \infty, \quad c_{0}\left|x^{j}-x^{0}\right| \leqslant \operatorname{dist}\left(x^{j}, D_{1}\right),
$$


for some $c_{0}>0$, where the last condition means nontangential approach of $x^{j}$ to $x^{0}$. Obviously it suffices to show that for any such sequence $\left\{x^{j}\right\}$ we have

$$
\left|\nabla u\left(y^{j}\right)\right| \rightarrow \infty \quad \forall y^{j} \in B\left(x^{j}, d_{j}\right),
$$

where $8 d_{j}=\operatorname{dist}\left(x^{j}, D_{1}\right)$. To show this, we scale the function $u$ by

$$
u_{j}(x):=\frac{u\left(d_{j} x+x^{j}\right)}{u\left(x^{j}\right)} \quad \text { in } B_{8}(0) .
$$

Since

$$
\Delta_{p} u_{j}=0, \quad u_{j}>0 \quad \text { in } B_{8}(0),
$$

we have, by Harnack's inequality,

$$
\sup _{B_{4}} u_{j} \leqslant C \inf _{B_{4}} u_{j} \leqslant C u_{j}(0)=C .
$$

In particular, $u_{j}$ is a bounded sequence in $B_{4}$. Hence by standard elliptic theory, a subsequence of $u_{j}$ converges to a solution $u_{0}$ in $B_{4}$, satisfying

$$
\Delta_{p} u_{0}=0, \quad u_{0}(0)=1, \quad u_{0}>0 \quad \text { in } B_{4} .
$$

Moreover, the level sets of $u_{0}$ are convex, since they are convex for all $u_{j}$.

Now suppose $\left|\nabla u\left(x^{j}\right)\right|>j$. Then by uniform $C^{1, \alpha}$ estimates

$$
C_{0} \geqslant\left|\nabla u_{j}(0)\right|=\frac{d_{j}\left|\nabla u\left(x^{j}\right)\right|}{u\left(x^{j}\right)} \geqslant \frac{j d_{j}}{u\left(x^{j}\right)} .
$$

Hence

$$
u\left(x^{j}\right) \geqslant \frac{j d_{j}}{C_{0}} .
$$

Now if for some

$$
y^{j}=d_{j} \tilde{y}^{j}+x^{j} \in B\left(x^{j}, d_{j}\right) \quad\left(\tilde{y}^{j} \in B_{1}\right),
$$

we have $\left|\nabla u\left(y^{j}\right)\right| \leqslant C_{1}$ for some $C_{1}>0$, then

$$
\left|\nabla u_{j}\left(\tilde{y}^{j}\right)\right|=\frac{d_{j}\left|\nabla u\left(d_{j} \tilde{y}^{j}+x^{j}\right)\right|}{u\left(x^{j}\right)}=\frac{d_{j}\left|\nabla u\left(y^{j}\right)\right|}{u\left(x^{j}\right)} \leqslant \frac{d_{j} C_{1}}{u\left(x^{j}\right)} \leqslant \frac{C_{1} C_{0}}{j},
$$

where in the last inequality we have used 177 . Hence it follows that $\left|\nabla u_{0}(\tilde{y})\right|=0$, where $\tilde{y}=$ $\lim \tilde{y}^{j} \in B_{1}$, for an appropriate subsequence.

To summarize, we have a positive $p$-harmonic function $u_{0}$ in $B_{4}$, with convex level sets, and with the further property that $\nabla u_{0}(\tilde{y})=0$ for some $\tilde{y} \in B_{1}$. This contradicts Hopf's boundary point lemma (see [17]), and completes the proof in this case.

The second case $x^{0} \in \partial D_{2}$ is treated similarly, with reversed argument. We sketch some details. We may start as in the previous case, assuming now

$$
\left|\nabla u\left(x^{j}\right)\right|<1 / j \quad \text { and } \quad\left|\nabla u\left(y^{j}\right)\right| \geqslant C_{0}>0,
$$


with $y^{j}$ as before

$$
y^{j}=d_{j} \tilde{y}^{j}+x^{j} \in B\left(x^{j}, d_{j}\right) \quad\left(\tilde{y}^{j} \in B_{1}\right) .
$$

Again all the above arguments are in order and we have the limit function $u_{0}$ and the limit point $\tilde{y}$ in $B_{1}$. Let us see what more information we can deduce. Indeed, on the one hand, by elliptic estimates,

and on the other hand

$$
C_{1} \geqslant\left|\nabla u_{j}\left(\tilde{y}^{j}\right)\right|=\frac{d_{j}\left|\nabla u\left(y^{j}\right)\right|}{u\left(x^{j}\right)} \geqslant \frac{d_{j} C_{0}}{u\left(x^{j}\right)},
$$

$$
\left|\nabla u_{j}(0)\right|=\frac{d_{j}\left|\nabla u\left(x^{j}\right)\right|}{u\left(x^{j}\right)} \leqslant \frac{d_{j}}{j u\left(x^{j}\right)} .
$$

Upon combining these estimates, we arrive at

$$
\left|\nabla u_{j}(0)\right| \leqslant \frac{C_{0} C_{1}}{j} .
$$

As $j$ tends to infinity we will have $\left|\nabla u_{0}(0)\right|=0$, and again Hopf's principle is violated.

The lower and upper semicontinuity follow in the same way as in the proof of Theorem 1.3 in [11].

LEMMA 3.8 Let $u$ be a solution to $\Delta_{p} u=0$ in a domain $\Omega$, and introduce the linear elliptic operator $L_{u}$ defined everywhere, except at critical points of $u$, by

$$
L_{u}:=|\nabla u|^{p-2} \Delta+(p-2)|\nabla u|^{p-4} \sum_{k, l=1}^{N} \frac{\partial u}{\partial x_{k}} \frac{\partial u}{\partial x_{l}} \frac{\partial^{2}}{\partial x_{k} \partial x_{l}} .
$$

Then $L_{u}\left(|\nabla u|^{p}\right) \geqslant 0$ in $\Omega$.

This lemma is essentially proved, though stated differently, in the papers of Payne and Philippin, [15] and [16]; see also the discussion in [9].

For two nested convex sets $D_{1} \subset D_{2}$, and for $x \in \partial D_{1}$, we denote by $T_{x, a}$ the supporting hyperplane at $x$ with the normal $a$ pointing away from $D_{1}$. Obviously, $T_{x, a}$ is not necessarily unique, depending on the geometry of $\partial D_{1}$. Now to each $x \in \partial D_{1}$ there corresponds a point $y_{x}$ (not necessarily unique) on $\partial D_{2} \cap\{z: a \cdot(z-x)>0\}$ such that $a \cdot\left(y_{x}-x\right)=\max a \cdot(z-x)$, where the maximum is taken over all $z \in \partial D_{2} \cap\{z: a \cdot(z-x)>0\}$.

LEMma 3.9 Let $D_{1}$ and $D_{2}$ be two nested convex domains $\left(\bar{D}_{1} \subset D_{2}\right)$ and denote by $u$ the p-capacitary potential of $D_{2} \backslash D_{1}$, i.e. the solution of

$$
\begin{cases}\Delta_{p} u=0 & \text { in } D_{2} \backslash \bar{D}_{1} \\ u=c_{1} & \text { on } \partial D_{1} \\ u=c_{2} & \text { on } \partial D_{2}\end{cases}
$$

where $c_{1}$ and $c_{2}$ are two given constants with $c_{1}>c_{2} \geqslant 0$. Then

$$
\limsup _{\substack{z \rightarrow x \\ z \in D_{2} \backslash \bar{D}_{1}}}|\nabla u(z)| \geqslant \limsup _{\substack{z \rightarrow y_{x} \\ z \in D_{2} \backslash \bar{D}_{1}}}|\nabla u(z)| \quad \forall x \in \partial D_{1},
$$

where $y_{x}$ is the point indicated in the discussion preceding this lemma.

For a proof of this lemma see [9], [10]. 
Definition 3.10 (extremal points) For a domain $D \in \mathbb{R}^{N}$ we say a point $x \in \partial D$ is an extremal point if there exists a supporting plane to $D$ touching $\partial D$ at $x$ only. We denote the set of all extremal points of $D$ by $E_{D}$.

LEMma 3.11 Retain the hypotheses in Lemma 3.9 and suppose also that $\partial D_{1}$ and $\partial D_{2}$ are $C^{1}$. Then

$$
|\nabla u(x)| \geqslant \inf _{y \in E_{D_{2}}}|\nabla u(y)| \text { for all } x \in D_{2} \backslash \bar{D}_{1} .
$$

This lemma is a consequence of Lemma 3.9 and geometric considerations.

The next lemma was an important tool in the variational existence treatment of the multi-layer problem by P. Laurence and E. Stredulinsky [13]:

Lemma 3.12 (see [13, Lemma 4.1], and [4, Thm. 1]) Retain the hypotheses in Lemma 3.9 Suppose moreover $\partial D_{i}(i=1,2)$ contains a line segment $l_{i}$, and that $|\nabla u| \geqslant c_{0}>0$. Then $|\nabla u|^{-1}$ is convex on $l_{2}$ and it is concave on $l_{1}$.

\section{The two-layer problem}

\subsection{Main result}

Let us consider two bounded convex domains $K_{1}$ and $K_{3}$ in $\mathbb{R}^{N}$ such that $K_{3}$ strictly contains $K_{1}$ (i.e. $K_{1} \subset \subset K_{3}$ ). We look for a convex domain $K_{2}$ such that

$$
K_{1} \subset \subset K_{2} \subset \subset K_{3}
$$

and the $p$-capacitary potentials $u_{1}$ and $u_{2}$ of the sets $K_{2} \backslash \bar{K}_{1}$ and $K_{3} \backslash \bar{K}_{2}$ respectively, i.e. solutions of

$$
\left\{\begin{array} { l l } 
{ \Delta _ { p } u _ { 1 } = 0 } & { \text { in } K _ { 2 } \backslash \overline { K } _ { 1 } , } \\
{ u _ { 1 } = 1 } & { \text { on } \partial K _ { 1 } , } \\
{ u _ { 1 } = 0 } & { \text { on } \partial K _ { 2 } , }
\end{array} \quad \left\{\begin{array}{ll}
\Delta_{p} u_{2}=0 & \text { in } K_{3} \backslash \bar{K}_{2}, \\
u_{2}=-1 & \text { on } \partial K_{3}, \\
u_{2}=0 & \text { on } \partial K_{2},
\end{array}\right.\right.
$$

satisfy a nonlinear joining condition like

$$
\left|\nabla u_{1}(x)\right|=g\left(x,\left|\nabla u_{2}(x)\right|\right) \quad \text { on } \partial K_{2} .
$$

We have the following result.

THEOREM 4.1 (two phases) Let $K_{1}, K_{3}$ be two convex domains such that $K_{3}$ strictly contains $K_{1}$, and $g \in \mathcal{G}$. Then there exists a convex $C^{1}$ domain $\omega, K_{1} \subset \subset \omega \subset \subset K_{3}$, which is a classical solution of the two-layer free boundary problem. The latter means that the $p$-capacitary potentials $u_{1}$ and $u_{2}$ of the sets $\omega \backslash \bar{K}_{1}$ and $K_{3} \backslash \bar{\omega}$ respectively (i.e. solutions of 20 with $K_{2}=\omega$ ) satisfy

$$
\lim _{\substack{z \rightarrow x \\ z \in \omega \backslash K_{1}}}\left|\nabla u_{1}(z)\right|=\lim _{\substack{y \rightarrow x \\ y \in K_{3} \backslash \omega}} g\left(y,\left|\nabla u_{2}(y)\right|\right) \quad \forall x \in \partial \omega .
$$

\subsection{Notations, definitions}

4.2.1 p-capacitary potentials. For every subdomain $\omega$ such that $K_{1} \subset \subset \omega \subset \subset K_{3}$, we set $\omega_{1}=\omega \backslash \bar{K}_{1}$ and $\omega_{2}=K_{3} \backslash \bar{\omega}$. We introduce the $p$-capacitary potentials $u_{1}^{\omega}$ (respectively $u_{2}^{\omega}$ ), or 
more simply $u_{1}$ (respectively $u_{2}$ ) when there is no possible confusion, which are the solutions of the boundary value problems

$$
\left\{\begin{array} { l l } 
{ \Delta _ { p } u _ { 1 } = 0 } & { \text { in } \omega _ { 1 } , } \\
{ u _ { 1 } = 1 } & { \text { on } \partial K _ { 1 } , } \\
{ u _ { 1 } = 0 } & { \text { on } \partial \omega , }
\end{array} \quad \left\{\begin{array}{ll}
\Delta_{p} u_{2}=0 & \text { in } \omega_{2} \\
u_{2}=-1 & \text { on } \partial K_{3} \\
u_{2}=0 & \text { on } \partial \omega
\end{array}\right.\right.
$$

In what follows, we will refer to $u_{1}$ as the inner potential and to $u_{2}$ as the outer potential of the set $\omega$. We want to find a domain $\Omega$ satisfying a joining condition

$$
\left|\nabla u_{1}(x)\right|=g\left(x,\left|\nabla u_{2}(x)\right|\right)
$$

as explained in the previous subsection. For that purpose, we introduce the following classes of domains:

4.2.2 Subsolutions, supersolutions. An open set $\omega$ (such that $K_{1} \subset \subset \omega \subset \subset K_{3}$ ) is called a subsolution (of the problem) if its $p$-capacitary potentials $u_{1}$ and $u_{2}$ satisfy

$$
\liminf _{\substack{z \rightarrow x \\ z \in \omega_{1}}}\left|\nabla u_{1}(z)\right| \geqslant \limsup _{\substack{y \rightarrow x \\ y \in \omega_{2}}} g\left(y,\left|\nabla u_{2}(y)\right|\right) \quad \forall x \in \partial \omega .
$$

An open set $\omega$ (such that $K_{1} \subset \subset \omega \subset \subset K_{3}$ ) is called a supersolution (of the problem) if its $p$-capacitary potentials $u_{1}$ and $u_{2}$ satisfy

$$
\limsup _{\substack{z \rightarrow x \\ z \in \omega_{1}}}\left|\nabla u_{1}(z)\right| \leqslant \liminf _{\substack{y \rightarrow x \\ y \in \omega_{2}}} g\left(y,\left|\nabla u_{2}(y)\right|\right) \quad \forall x \in \partial \omega .
$$

4.2.3 The classes $\mathcal{A}, \mathcal{B}, \mathcal{C}$. We are going to work only with convex domains, so let us set

$$
\mathcal{C}=\left\{\omega \text { convex bounded open subset of } \mathbb{R}^{N}: K_{1} \subset \subset \omega \subset \subset K_{3}\right\} .
$$

We denote by $\mathcal{A}$ the class of convex subsolutions and $\mathcal{B}$ the class of convex supersolutions:

$$
\begin{gathered}
\mathcal{A}=\left\{\omega \in \mathcal{C}: \liminf _{\substack{z \rightarrow x \\
z \in \omega_{1}}}\left|\nabla u_{1}(z)\right| \geqslant \limsup _{\substack{y \rightarrow x \\
y \in \omega_{2}}} g\left(y,\left|\nabla u_{2}(y)\right|\right) \forall x \in \partial \omega\right\}, \\
\mathcal{B}=\left\{\omega \in \mathcal{C}: \limsup _{\substack{z \rightarrow x \\
z \in \omega_{1}}}\left|\nabla u_{1}(z)\right| \leqslant \liminf _{\substack{y \rightarrow x \\
y \in \omega_{2}}} g\left(y,\left|\nabla u_{2}(y)\right|\right) \forall x \in \partial \omega\right\} .
\end{gathered}
$$

A classical solution of the two-phase free boundary problem is obviously a domain $\Omega \in \mathcal{A} \cap \mathcal{B}$.

\subsection{Stability results for the class $\mathcal{B}$}

First we show that the class $\mathcal{B}$ is closed under intersection.

Lemma 4.2 Let $\omega^{1}, \omega^{2}$ be in $\mathcal{B}$. Then $\omega^{1} \cap \omega^{2} \in \mathcal{B}$. 
Proof. As the intersection of two convex domains is convex, we need to prove the condition on the gradients for $u_{1}:=u_{1}^{\omega^{1} \cap \omega^{2}}$ and $u_{2}:=u_{2}^{\omega^{1} \cap \omega^{2}}$ at the boundary of $\omega^{1} \cap \omega^{2}$. By the comparison principle, $0 \leqslant u_{1} \leqslant \min \left(u_{1}^{\omega^{1}}, u_{1}^{\omega^{2}}\right)$, which implies that for $x \in \partial\left(\omega^{1} \cap \omega^{2}\right) \subset \partial \omega^{1} \cup \partial \omega^{2}$ we have (for example, we choose the case where $x \in \partial \omega^{1}$ )

$$
u_{1}(x)=u_{1}^{\omega^{1}}(x)=0 \text { and } \limsup _{\substack{y \rightarrow x \\ y \in\left(\omega^{1} \cap \omega^{2}\right)_{1}}}\left|\nabla u_{1}(y)\right| \leqslant \limsup _{\substack{y \rightarrow x \\ y \in \omega_{1}^{1}}}\left|\nabla u_{1}^{\omega^{1}}(y)\right|,
$$

while, since $u_{2} \leqslant \min \left(u_{2}^{\omega^{1}}, u_{2}^{\omega^{2}}\right) \leqslant 0$, we have

$$
u_{2}(x)=u_{2}^{\omega^{1}}(x)=0 \text { and } \liminf _{\substack{y \rightarrow x \\ y \in\left(\omega^{1} \cap \omega^{2}\right)_{2}}}\left|\nabla u_{2}(y)\right| \geqslant \liminf _{\substack{y \rightarrow x \\ y \in \omega_{2}^{1}}}\left|\nabla u_{2}^{\omega^{1}}(y)\right| .
$$

Now, by monotonicity of $g$ with respect to its second argument, and the fact that $\omega^{1}$ belongs to $\mathcal{B}$,

$$
\begin{gathered}
\limsup _{\substack{y \rightarrow x \\
y \in\left(\omega^{1} \cap \omega^{2}\right)_{1}}}\left|\nabla u_{1}(y)\right| \leqslant \limsup _{\substack{y \rightarrow x \\
y \in \omega_{1}^{1}}}\left|\nabla u_{1}^{\omega^{1}}(y)\right| \leqslant \liminf _{\substack{y \rightarrow x \\
y \in \omega_{2}^{1}}} g\left(y,\left|\nabla u_{2}(y)\right|\right) \\
\leqslant \liminf _{\substack{y \rightarrow x \\
y \in\left(\omega^{1} \cap \omega^{2}\right)_{2}}} g\left(y,\left|\nabla u_{2}(y)\right|\right) .
\end{gathered}
$$

Now, the technical and more difficult point is to prove that $\mathcal{B}$ is stable, in some sense, for decreasing sequences of convex domains. Indeed, our aim is to construct a solution to the free boundary problem by taking a minimal element (for inclusion) in the class $\mathcal{B}$. So, we need some stability of $\mathcal{B}$ under the constructing process that we are going to use.

THEOREM 4.3 Let $\omega^{1} \supset \omega^{2} \supset \cdots$ be a decreasing sequence of convex domains in $\mathcal{B}$, and suppose $\omega=\overline{\bigcap \omega^{n}}$ (the interior of the closure) belongs to $\mathcal{C}$. Then $\omega \in \mathcal{B}$.

Proof. Since the domains involved are convex and they all contain $K_{1}$, they are uniformly Lipschitz. In particular, by standard regularity up to boundary (see $[\mathrm{K}]$ ), the $p$-capacitary potentials $u_{1}^{n}, u_{2}^{n}$ are $C^{\alpha}$ ( $\alpha$ depending on the uniform cone property of $\omega^{n}$ ) in the entire space $\mathbb{R}^{N}$ (after appropriate extension). Since also $u_{1}^{n}, u_{2}^{n}$ are decreasing sequences we have limit functions $u_{1}, u_{2}$ which are the $p$-capacitary potentials of $\omega_{1}=\omega \backslash K_{1}$, and $\omega_{2}=K_{3} \backslash \bar{\omega}$, respectively. Moreover, by local $C^{1, \alpha}$ regularity (see [14]), convergence takes place also for the gradients on every compact subset of $\omega_{1}$ and $\omega_{2}$ respectively.

We need to show $\omega \in \mathcal{B}$. Let $\epsilon>0$ be small enough and fix $x^{0} \in K_{1}$. Now for each $y \in \partial \omega$ let us denote by $R\left(x^{0}, y\right)$ the ray emanating from $x^{0}$ and traveling through $y$. Then, by the choice of $x^{0}$, and the convexity of the sets $\omega, \omega^{n}$ we can choose unique points $x=x(y, n) \in \partial \omega^{n} \cap R\left(x^{0}, y\right)$ and $x^{\epsilon} \in\left\{u_{2}^{n}=-\delta_{\epsilon}\right\} \cap R\left(x^{0}, y\right)$, where $\delta_{\epsilon}>0$ is to be chosen later. It follows that

$$
\lim _{n \rightarrow \infty} x(y, n)=y, \quad \lim _{\epsilon \rightarrow 0} x^{\epsilon}=x(y, n) \quad \text { nontangentially. }
$$

Next denote by $v_{n}$ the solution of the following boundary value problem:

$$
\begin{cases}L_{u_{1}^{n}}\left(v_{n}\right)=0 & \text { in }\left\{0<u_{1}^{n}<1 / 2\right\} \\ v_{n}(x)=\left|\nabla u_{1}^{n}(x)\right|^{p} & \text { on }\left\{u_{1}^{n}=1 / 2\right\} \\ v_{n}(x)=G_{n, \epsilon}(x) & \text { on } \partial \omega^{n}\end{cases}
$$


where

$$
G_{n, \epsilon}(x)=\min \left(2 M^{p}, g\left(x,\left|\nabla u_{2}^{n}\left(x^{\epsilon}\right)\right|+\epsilon\right)^{p}\right), \quad \text { with } M=\sup _{\left\{0<u_{1}^{n}<1 / 2\right\}}\left|\nabla u_{1}^{n}\right|,
$$

and $L_{u_{1}^{n}}$ is defined in Lemma 3.8 Observe that the boundedness of $M$ follows by simple (linear) barrier argument.

Fix a point $y \in \partial \omega$. Then two possibilities may arise (see Lemma 3.7):

Case 1: $\left|\nabla u_{2}(y)\right|=\infty$ nontangentially,

Case 2: $\left|\nabla u_{2}(y)\right|=M_{1}$ nontangentially.

In both cases we will have

$$
\left|\nabla u_{2}^{n}\left(x^{\epsilon}\right)\right| \approx\left|\nabla u_{2}^{n}(x)\right|
$$

In Case 1 we obtain

$$
g\left(x,\left|\nabla u_{2}^{n}\left(x^{\epsilon}\right)\right|+\epsilon\right)^{p}>2 M^{p} \geqslant\left|\nabla u_{1}^{n}(x)\right|^{p},
$$

i.e., $G_{n, \epsilon}(x) \geqslant\left|\nabla u_{1}^{n}(x)\right|^{p}$.

In Case 2 we have (by nontangential continuity of $\left|\nabla u_{2}^{n}\right|$ )

$$
\left|\nabla u_{2}^{n}(x)\right| \leqslant\left|\nabla u_{2}^{n}\left(x^{\epsilon}\right)\right|+\epsilon,
$$

provided $\delta_{\epsilon}$ is small enough. And by nondecreasing property of $g$ we have

$$
g\left(x,\left|\nabla u_{2}^{n}\left(x^{\epsilon}\right)\right|+\epsilon\right)^{p} \geqslant g\left(x,\left|\nabla u_{2}^{n}(x)\right|\right)^{p} \geqslant\left|\nabla u_{1}^{n}(x)\right|^{p},
$$

Hence

$$
G_{n, \epsilon}(x) \geqslant\left|\nabla u_{1}^{n}(x)\right|^{p}
$$

Therefore upon applying the comparison principle (for the operator $L_{u_{1}^{n}}$; see Lemma 3.8 we can obtain

$$
v_{n}(x) \geqslant\left|\nabla u_{1}^{n}(x)\right|^{p} \quad \text { in }\left\{0<u_{1}^{n}<1 / 2\right\} \text {. }
$$

Now as $n \rightarrow \infty$,

$$
v(x):=\lim _{n} v_{n}(x) \geqslant\left|\nabla u_{1}(x)\right|^{p} \quad \text { in } \quad\left\{0<u_{1}<1 / 2\right\} .
$$

Since $x^{\epsilon}$ is compactly inside $\omega_{2}$ and $\nabla u_{2}^{n}\left(x^{\epsilon}\right) \rightarrow \nabla u_{2}\left(x^{\epsilon}\right)$ in $C^{\alpha}$-norm (see [14]) we have a uniform convergence of

$$
\left.v_{n}\right|_{\partial \omega_{1}^{n}}=G_{n, \epsilon}(x)=\min \left(2 M^{p}, g\left(x,\left|\nabla u_{2}^{n}\left(x^{\epsilon}\right)\right|+\epsilon\right)^{p}\right)
$$

to

$$
G_{\epsilon}(x)=\min \left(2 M^{p}, g\left(x,\left|\nabla u_{2}\left(x^{\epsilon}\right)\right|+\epsilon\right)^{p}\right) .
$$

Therefore for $z \in B\left(x, r_{\epsilon}\right) \cap \omega_{1}$ and $x \in \partial \omega$,

$$
\left|\nabla u_{1}(z)\right|^{p} \leqslant v(z) \leqslant G_{\epsilon}(x)+\epsilon \leqslant g\left(x,\left|\nabla u_{2}\left(x^{\epsilon}\right)\right|+\epsilon\right)^{p}+\epsilon,
$$

provided $r_{\epsilon}$ is small enough. By Lemma 3.7, and continuity of $g$ (as $\epsilon \rightarrow 0$ ), we get

$$
\underset{\substack{z \rightarrow x \\ z \in \omega_{1}}}{\limsup }\left|\nabla u_{1}(z)\right| \leqslant \liminf _{\substack{y \rightarrow x \\ y \in \omega_{2}}} g\left(y,\left|\nabla u_{2}(y)\right|\right) .
$$

Hence $\omega \in \mathcal{B}$. 


\subsection{Proof of Theorem 4.1}

Step 1: Existence of subsolutions and supersolutions. Let us consider the solution $u$ of the boundary value problem ( $p$-capacitary potential)

$$
\begin{cases}\Delta_{p} u=0 & \text { in } K_{3} \backslash \bar{K}_{1} \\ u=1 & \text { on } K_{1} \\ u=-1 & \text { on } \overline{\mathbb{R}^{N} \backslash K_{3}}\end{cases}
$$

For any $-1<\alpha<1$, let $\omega_{\alpha}=\{u(x)>\alpha\}$. Also define $u_{1, \alpha}(x)=(u(x)-\alpha) /(1-\alpha)$ in the closure of $\omega_{1, \alpha}=\{\alpha<u(x)<1\}$ and $u_{2, \alpha}(x)=(u(x)-\alpha) /(1+\alpha)$ in the closure of $\omega_{2, \alpha}=\{-1<u(x)<\alpha\}$. Then $\omega_{\alpha}$ is a supersolution (resp. subsolution) if

$$
\frac{|\nabla u(x)|}{1-\alpha}=\left|\nabla u_{1, \alpha}(x)\right|<(>) g\left(x,\left|\nabla u_{2, \alpha}(x)\right|\right)=g\left(x, \frac{|\nabla u(x)|}{1+\alpha}\right)
$$

for all $x \in \partial \omega_{\alpha}$. But a comparison argument involving the $p$-capacitary potential in any slab between parallel boundary planes tangent to $\partial \omega_{\alpha}$ and $\partial K_{3}$ shows that $|\nabla u(x)| \geqslant(\alpha+1) / M \geqslant(1+\alpha) / R$ for all $x \in \partial \omega_{\alpha}$, where $M=\sup \left\{\operatorname{dist}\left(x, \omega_{\alpha}\right): x \in \partial K_{3}\right\}$. Therefore, $\omega_{\alpha}$ is a supersolution (resp. subsolution) provided that

$$
\frac{1+\alpha}{1-\alpha}<(>) \frac{g(x, y)}{y}
$$

for all $x \in \partial \omega_{\alpha}$ and all $y \geqslant y_{0}=1 / R$. Applying Assumption (A4), we see that $\omega_{\alpha}$ is a supersolution if $(1+\alpha) /(1-\alpha) \leqslant C_{1}$ (true for $\alpha$ sufficiently close to -1$)$, and that $\omega_{\alpha}$ is a subsolution if $(1+\alpha) /(1-\alpha) \geqslant C_{2}$ (true for $\alpha$ sufficiently close to 1 ).

We remark that $K_{1}$ and $K_{3}$ are regular, so that $|\nabla u(x)|$ is both uniformly bounded and uniformly positive in $K_{3} \backslash \bar{K}_{1}$. Then the above argument yields supersolutions and subsolutions without involving Assumption (A4) (one can replace it by the much weaker assumption that $g(x, y) \rightarrow \infty$ as $y \rightarrow \infty$ uniformly over $x \in K$ ).

In what follows, $\Omega_{0}$ will denote a given subsolution and $\Omega_{1}$ a given supersolution.

Step 2: Construction of a minimal element in the class $\mathcal{B}$. We introduce the class $\mathcal{S}:=\{\omega \in \mathcal{B}$ : $\Omega_{0} \subset \omega \subset \Omega_{1}$ \}. Let $I$ be the intersection of all domains in the class $\mathcal{S}$ and set $\Omega=\bar{I}$ (the interior of the closure, which is still convex). To prove $\Omega \in \mathcal{B}$, we select a sequence $\left\{\omega_{n}\right\}_{n=1}^{\infty}$ of domains in $\mathcal{S}$ such that $\bigcap_{n \geqslant 1} \omega_{n}=I$ and we consider the sequence $\left\{\Omega_{n}\right\}_{n=1}^{\infty}$ of domains defined by $\Omega_{1}=\omega_{1}$ and $\Omega_{n+1}=\Omega_{n} \cap \omega_{n+1}(n \geqslant 1)$. By Lemma 4.2 each $\Omega_{n}$ is convex and belongs to $\mathcal{B}$. Hence, since $\Omega_{i+1} \subset \Omega_{i}$, Theorem 4.3 gives the desired result.

Step 3: On $E_{\Omega}$, the extremal points of $\Omega$, we have $\lim \sup \left|\nabla u_{1}(z)\right|=\lim \inf g\left(y,\left|\nabla u_{2}(y)\right|\right)$. This property can be proved in the same way as in [9], but since it is slightly more complicated and for the sake of completeness, we give here the complete proof. Suppose the property fails. Then there exists $X_{0} \in E_{\Omega}$ such that

$$
\limsup _{\substack{z \rightarrow X_{0} \\ z \in \Omega_{1}}}\left|\nabla u_{1}(z)\right|=\liminf _{\substack{y \rightarrow X_{0} \\ y \in \Omega_{2}}} g\left(y,\left|\nabla u_{2}(y)\right|\right)(1-4 \alpha) \quad \text { with } \alpha>0 .
$$

We define

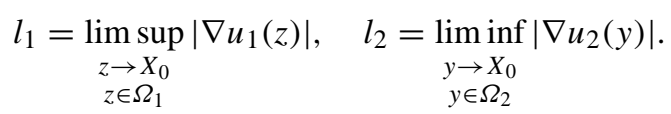


Note that since $g$ is continuous and nondecreasing, $\liminf _{y \rightarrow X_{0}} g\left(y,\left|\nabla u_{2}(y)\right|\right)=g\left(X_{0}, l_{2}\right)$. Therefore assumption 27) can be written as

$$
l_{1}=g\left(X_{0}, l_{2}\right)(1-4 \alpha)
$$

We assume first $l_{2}<\infty$. Hence for some small neighborhood $\mathcal{V}$ of $X_{0}$ we have

$$
\begin{array}{ll}
\left|\nabla u_{1}(z)\right| \leqslant l_{1}(1+\alpha) & \forall z \in \mathcal{V} \cap \Omega_{1}, \\
\left|\nabla u_{2}(y)\right| \geqslant l_{2}(1-\alpha) & \forall y \in \mathcal{V} \cap \Omega_{2} .
\end{array}
$$

Let us fix a hyperplane $T_{d}$, parallel to a supporting plane at $X_{0}$, with $\operatorname{dist}\left(X_{0}, T_{d}\right)=d$ and such that $T_{d} \cap \Omega \subset \mathcal{V}$. This is possible due to the extremal property of $X_{0}$.

By rotation and translation, we assume $X_{0}$ is the origin and $T_{d}=\left\{x_{1}=d\right\}$. Let $T_{\delta}=\left\{x_{1}=\delta\right\}$ and set $\Omega^{\delta}=\Omega \backslash\left\{x_{1} \leqslant \delta\right\}$. Then by the comparison principle the (inner) $p$-capacitary potential $u_{1}^{\delta}$ of $\Omega_{1}^{\delta}$ satisfies

$$
0 \leqslant u_{1}^{\delta} \leqslant u_{1} \quad \text { in } \Omega_{1}^{\delta},
$$

while the (outer) potential satisfies

$$
u_{2}^{\delta} \leqslant u_{2} \leqslant 0 \quad \text { in } \Omega_{2},
$$

which implies that for points $x$ belonging to $\partial \Omega \cap \partial \Omega_{\delta}$,

$$
\limsup _{\substack{y \rightarrow x \\ y \in \Omega_{1}^{\delta}}}\left|\nabla u_{1}^{\delta}(y)\right| \leqslant \limsup _{\substack{y \rightarrow x \\ y \in \Omega_{1}}}\left|\nabla u_{1}(y)\right| \leqslant \liminf _{\substack{y \rightarrow x \\ y \in \Omega_{2}}} g\left(y,\left|\nabla u_{2}(y)\right|\right) \leqslant \liminf _{\substack{y \rightarrow x \\ y \in \Omega_{2}^{\delta}}} g\left(y,\left|\nabla u_{2}^{\delta}(y)\right|\right) .
$$

Now by 29, and (31),

$$
\max _{T_{d}} u_{1}^{\delta} \leqslant \max _{T_{d}} u_{1} \leqslant d \sup _{\left\{0 \leqslant x_{1} \leqslant d\right\}}\left|\nabla u_{1}\right| \leqslant l_{1}(1+\alpha) d .
$$

Define

$$
v:=u_{1}^{\delta}+\frac{l_{1}(1+\alpha) d}{d-\delta}\left(d-x_{1}\right) .
$$

Since the second derivatives of $v$ and $u_{1}^{\delta}$ coincide, we have

$$
L_{u_{1}^{\delta}} v=L_{u_{1}^{\delta}} u_{1}^{\delta}=0 \quad \text { in } \Omega_{1}^{\delta} \cap\left\{x_{1}<d\right\} .
$$

Therefore in $\Omega_{1}^{\delta} \cap\left\{x_{1}<d\right\}$, $v$ takes its maximum on the boundary. By inspection and 33 , it is easy to see that on $\partial\left(\Omega_{\delta} \cap\left\{x_{1}<d\right\}\right) \subset T_{d} \cup T_{\delta} \cup\left(\partial \Omega \cap\left\{\delta<x_{1}<d\right\}\right)$,

$$
v \leqslant l_{1}(1+\alpha) d
$$

with equality on $T_{\delta}$. Hence $\partial v / \partial x_{1} \leqslant 0$ on $T_{\delta}$, i.e.,

$$
\left|\nabla u_{1}^{\delta}\right| \leqslant \frac{l_{1}(1+\alpha) d}{d-\delta} \quad \text { on } T_{\delta}
$$


Now, it remains to estimate $\left|\nabla u_{2}^{\delta}\right|$ on $T_{\delta}$. For that purpose, let us introduce a part of a level set of $u_{2}^{\delta}$ contained in the neighborhood $\mathcal{V} \cap \Omega_{2}$ and consider, on that level set, one point, say $x_{\delta}$, where the supporting hyperplane is parallel to $T_{\delta}$. By Lemma 3.9, we have

$$
\forall y \in T_{\delta} \quad\left|\nabla u_{2}^{\delta}(y)\right| \geqslant\left|\nabla u_{2}^{\delta}\left(x_{\delta}\right)\right| .
$$

Now, by continuity of $g$, we can choose $\varepsilon$ and $\delta$ small enough such that

$$
\forall y \in T_{\delta} \quad g\left(X_{0}, l_{2}\right) \leqslant g\left(y, l_{2}(1-\varepsilon)\right)(1+\alpha) .
$$

Now, by uniform convergence of $\left|\nabla u_{2}^{\delta}\right|$ to $\left|\nabla u_{2}\right|$ when $\delta \rightarrow 0$ on the level set, we can choose $\delta$ small enough such that

$$
\left|\nabla u_{2}^{\delta}(y)\right| \geqslant\left|\nabla u_{2}^{\delta}\left(x_{\delta}\right)\right| \geqslant l_{2}(1-\varepsilon) .
$$

Replacing in (36) and using (28), (34) and the monotonicity of $g$ yields

$$
\forall y \in T_{\delta} \quad\left|\nabla u_{1}^{\delta}(y)\right| \leqslant \frac{g\left(y,\left|\nabla u_{2}^{\delta}(y)\right|\right)(1+\alpha)^{2}(1-4 \alpha) d}{d-\delta} .
$$

Now, it suffices to choose $\delta$ even smaller so that

$$
\frac{(1+\alpha)^{2}(1-4 \alpha) d}{d-\delta} \leqslant 1
$$

which in turn implies $\Omega_{\delta} \in \mathcal{B}$. Since $\Omega_{\delta} \subset \Omega$ we have reached a contradiction.

Now, if $l_{2}=+\infty$, we can choose the neighborhood $\mathcal{V}$ in such a way that

$$
\left|\nabla u_{2}(y)\right| \geqslant 2 M \quad \forall y \in \mathcal{V} \cap \Omega_{2},
$$

where $M=\sup _{x \in \Omega_{1}}\left|\nabla u_{1}(x)\right|$. Then we reach a contradiction in exactly the same way, by choosing $\delta$ small enough such that $\Omega_{\delta}$ will be in the class $\mathcal{B}$.

Step 4: The boundary of $\Omega$ is $C^{1}$. It suffices to show that at each boundary point there exists a unique tangent plane. Suppose the latter fails. Let $x^{0} \in \partial \Omega$ with two supporting planes $\Pi_{1}, \Pi_{2}$ at $x^{0}$. Then by barrier arguments (Lemmas $3.1+3.2$

$$
\lim _{\Omega_{1} \ni y \rightarrow \partial \Omega \cap \Pi_{1} \cap \Pi_{2} .}\left|\nabla u_{1}(y)\right|=0 \quad \text { and } \quad \lim _{\Omega_{2} \ni z \rightarrow \partial \Omega \cap \Pi_{1} \cap \Pi_{2} .}\left|\nabla u_{2}(z)\right|=+\infty .
$$

Let $\Pi_{3}$ be a third plane supporting $\partial \Omega$ at $x^{0}$ and such that $\Pi_{3} \cap \partial \Omega \subset \Pi_{1} \cap \Pi_{2}$, i.e., $\Pi_{3}$ does not touch any other boundary points of $\Omega$ than those in the intersection of the planes $\Pi_{1}$ and $\Pi_{2}$. Now, move $\Pi_{3}$ towards the interior of $\Omega$ so that it cuts off a small cap from $\Omega$; it may well be a tub-like region. Then a similar argument as in the previous step will prove that this new domain is still in the class $\mathcal{B}$. This contradicts the minimal property of $\Omega$.

Step 5: The nonlinear joining condition holds on $E_{\Omega}$. Let $x \in E_{\Omega}$ be fixed. On the one hand, we have the following chain of (in)equalities (here n.t. means nontangentially, see Lemma 3.7 for details):

$$
\begin{aligned}
\left|\nabla u_{1}(x)\right|: & =\lim _{\substack{z \rightarrow x \text { n.t. } \\
z \in \Omega_{1}}}\left|\nabla u_{1}(z)\right| \leqslant \limsup _{\substack{z \rightarrow x \\
z \in \Omega_{1}}}\left|\nabla u_{1}(z)\right| \\
& \leqslant \liminf _{\substack{y \rightarrow x \\
y \in \Omega_{2}}} g\left(y,\left|\nabla u_{2}(y)\right|\right) \leqslant \lim _{\substack{y \rightarrow x \text { n.t. } \\
y \in \Omega_{2}}} g\left(y,\left|\nabla u_{2}(y)\right|\right):=g\left(x,\left|\nabla u_{2}(x)\right|\right),
\end{aligned}
$$


where the first and last equalities are due to Lemma 3.7 the second and fourth inequalities come from the definition of liminf and limsup (we also use the continuity of $g$ ) and the third inequality comes from the fact that $\Omega$ belongs to the class $\mathcal{B}$. On the other hand, we have the following chain of (in)equalities:

$$
\left|\nabla u_{1}(x)\right| \geqslant \limsup _{\substack{z \rightarrow x \\ z \in \Omega_{1}}}\left|\nabla u_{1}(z)\right|=\liminf _{\substack{y \rightarrow x \\ y \in \Omega_{2}}} g\left(y,\left|\nabla u_{2}(y)\right|\right) \geqslant g\left(x,\left|\nabla u_{2}(x)\right|\right),
$$

where the first inequality is the upper semicontinuity of $u_{1}$ at $x$, the equality is step 3 , and the second inequality is the lower semicontinuity of $u_{2}$ at $x$. Now, (37) and (38) together give the desired result.

Step 6: The nonlinear joining condition holds at every boundary point. According to step 5, it remains to prove the equality $\left|\nabla u_{1}(x)\right|=g\left(x,\left|\nabla u_{2}(x)\right|\right)$ on maximal line segments in $I=[a, b] \subset$ $\partial \Omega$. For any such line segment one readily verifies that $a, b \in \bar{E}_{\Omega}$. Also at the points $a, b$ we have equation (38) verified. In view of assumption (A3) for the function $g$ in conjunction with Lemma 3.12 we claim that the function

$$
x \mapsto \frac{1}{\left|\nabla u_{1}(x)\right|}-\frac{1}{g\left(x,\left|\nabla u_{2}(x)\right|\right)}
$$

is convex, nonnegative. The latter depends on the fact that $\Omega$ belongs to the class $\mathcal{B}$ and it vanishes at the extremities of any segment (by step 5 and n.t.-continuity). Therefore, this function vanishes identically. This completes the proof.

\section{Uniform separation estimate}

TheOREM 5.1 (cf. [2, Lemma 4.4]) Let $H$ denote the set of all configurations $\left(K_{1}, \omega, K_{3}\right)$ such that $K_{1}, \omega, K_{3}$ are convex,

$$
B_{\rho}(0) \subset K_{1} \subset \subset \omega \subset \subset K_{3} \subset B_{R}(0),
$$

and $\omega$ is a supersolution relative to $K_{1}$ and $K_{3}$. Then there exists a value $\eta>0$ such that

$$
\operatorname{dist}\left(\partial K_{1}, \partial \omega\right) \geqslant \eta \operatorname{dist}\left(\partial K_{1}, \partial K_{3}\right)
$$

uniformly for all $\left(K_{1}, \omega, K_{3}\right) \in H$.

This result follows directly from Lemmas 5.2 and 5.3 below.

LEMMA 5.2 For any $\left(K_{1}, \omega, K_{3}\right) \in H$, let

$$
\alpha=\max \{u(x): x \in \partial \omega\} \in(-1,1),
$$

where $u$ solves the Dirichlet problem 26]. Then there exists a value $\alpha_{0} \in(-1,1)$ such that $\alpha \leqslant \alpha_{0}$ uniformly over all $\left(K_{1}, \omega, K_{3}\right) \in H$.

Proof. It suffices to consider only configurations in $H$ such that $\alpha \in(0,1)$. Given such a configuration (and the corresponding value $\alpha$ ), let $u_{1}, u_{2}, \omega_{1}, \omega_{2}$ be as defined in (23). Define the $p$-harmonic functions $u_{1, \alpha}(x)=(u(x)-\alpha) /(1-\alpha)$ and $u_{2, \alpha}(x)=(u(x)-\alpha) /(1+\alpha)$, both in the closure of the set $\Omega:=K_{3} \backslash \bar{K}_{1}$. Then $u_{1}=u_{1, \alpha}=1$ on $\partial K_{1}$ and $u_{1, \alpha} \leqslant 0=u_{1}$ on $\partial \omega$. 
It follows by the comparison principle for $p$-harmonic functions that $u_{1, \alpha} \leqslant u_{1}$ in $\omega_{1}$. Similarly, we have $u_{2}=u_{2, \alpha}=-1$ on $\partial K_{3}$ and $u_{2, \alpha} \leqslant 0=u_{2}$ on $\partial \omega$, from which it follows by the comparison principle that $u_{2, \alpha} \leqslant u_{2}$ in $\omega_{2}$. We choose a point $x_{0} \in \partial \omega$ such that $u\left(x_{0}\right)=\alpha$. Clearly the function $u$ is regular near $x_{0} \in \Omega$ (and therefore so are $u_{1, \alpha}$ and $u_{2, \alpha}$ ). For small $\delta>0$, let $x_{\delta}=x_{0}+\delta v_{0} \in \omega_{1}$, where $v_{0}$ denotes the unit vector with direction opposite $\nabla u\left(x_{0}\right)$. Also let $\gamma_{\delta} \subset \omega_{1}$ denote the directed line segment of length $\delta$ joining $x_{0}$ to $x_{\delta}$. Clearly

$$
\partial u_{1}(x) / \partial \nu_{0} \leqslant\left|\nabla u_{1}(x)\right| \leqslant \sup _{x \in \gamma_{\delta}}\left|\nabla u_{1}(x)\right|
$$

and

$$
\left|\partial u_{1, \alpha}(x) / \partial v_{0}-\right| \nabla u_{1, \alpha}\left(x_{0}\right)|| \leqslant z(\delta),
$$

both on $\gamma_{\delta}$, where $z(\delta) \rightarrow 0$ as $\delta \rightarrow 0$. Therefore

$$
\begin{aligned}
0 & \leqslant u_{1}\left(x_{\delta}\right)-u_{1, \alpha}\left(x_{\delta}\right)=\int_{\gamma_{\delta}}\left(\partial / \partial \nu_{0}\right)\left(u_{1}(x)-u_{1, \alpha}(x)\right) d s \\
& \left.\leqslant \sup _{x \in \gamma_{\delta}}\left|\nabla u_{1}(x)\right|-\left|u_{1, \alpha}\left(x_{0}\right)\right|+z(\delta)\right) \delta,
\end{aligned}
$$

from which it follows that

$$
\limsup _{\omega_{1} \ni x \rightarrow x_{0}}\left|\nabla u_{1}(x)\right| \geqslant\left|\nabla u_{1, \alpha}\left(x_{0}\right)\right|=\frac{\left|\nabla u\left(x_{0}\right)\right|}{1-\alpha} .
$$

For small $\delta>0$, let $\gamma_{\delta}$ denote a directed arc of steepest ascent of $u_{2}$ of length $\delta$, joining a point $x_{\delta} \in \omega_{2}$ to the point $x_{0}$. Since $\partial u_{2}(x) / \partial v=\left|\nabla u_{2}(x)\right|$ on $\gamma_{\delta}$, where $v$ denotes the forward unit tangent vector to the arc, we have

$$
0 \geqslant u_{2, \alpha}\left(x_{\delta}\right)-u_{2}\left(x_{\delta}\right)=\int_{\gamma_{\delta}}(\partial / \partial \nu)\left(u_{2}(x)-u_{2, \alpha}(x)\right) d s \geqslant \int_{\gamma_{\delta}}\left(\left|\nabla u_{2}(x)\right|-\left|\nabla u_{2, \alpha}(x)\right|\right) d s,
$$

from which it follows that

$$
\inf _{x \in \gamma_{\delta}}\left|\nabla u_{2}(x)\right| \leqslant\left|\nabla u_{2, \alpha}\left(x_{0}\right)\right|+z(\delta)
$$

and therefore that

$$
\liminf _{\omega_{2} \ni x \rightarrow x_{0}}\left|\nabla u_{2}(x)\right| \leqslant\left|\nabla u_{2, \alpha}\left(x_{0}\right)\right|=\frac{\left|\nabla u\left(x_{0}\right)\right|}{1+\alpha} .
$$

In view of the definition of an exterior solution, it follows from (40) and (41) that

$$
\left|\nabla u\left(x_{0}\right)\right| /(1-\alpha) \leqslant g\left(x_{0},\left|\nabla u\left(x_{0}\right)\right| /(1+\alpha)\right) .
$$

A simple comparison argument involving the $p$-capacitary potential in a slab bounded by parallel planes, one tangent to the surface $\{u(x)=\alpha\}$ at $x_{0}$, the other tangent to $\partial K_{3}$, shows that $\left|\nabla u\left(x_{0}\right)\right| \geqslant$ $(\alpha+1) / M \geqslant(\alpha+1) / R$, where $M=\sup _{x \in \partial K_{3}} \operatorname{dist}(x,\{u(x)=\alpha\})$. It follows from (42) and Assumption (A4) that

$$
1 /(1-\alpha) \leqslant(1+\alpha) /(1-\alpha) \leqslant g\left(x_{0}, y\right) / y \leqslant C_{2},
$$

where we set $y=\left|\nabla u\left(x_{0}\right)\right| /(1+\alpha) \geqslant y_{0}=1 / R$, and where $C_{2}$ depends only on $R, y_{0}$, and the function $g$. The assertion follows, since (43) cannot be satisfied unless $\alpha \leqslant \alpha_{0}=1-1 / C_{2}$. 
Lemma 5.3 In the context of Lemma 5.2 , there is a constant $\eta>0$ such that

$$
\operatorname{dist}\left(\partial K_{1},\left\{u(x)=\alpha_{0}\right\}\right) \geqslant \eta \operatorname{dist}\left(\partial K_{1}, \partial K_{3}\right)
$$

for any convex sets $K_{1}, K_{3}$ such that $B_{\rho}(0) \subset K_{1} \subset \subset K_{3} \subset B_{R}(0)$.

Proof. For any $r \in(0,1]$ and unit vector $v$, let $E(r, v)=\left\{x \in \mathbb{R}^{N}: \operatorname{dist}(x, D(r, v))<r\right\}$, where $\lambda=\rho^{2} / R$ and $D(r, v)$ denotes the closure of the convex hull of the set $\{0\} \cup B_{\lambda r}(-r \rho v)$. Let $u_{r, v}(x)$ denote the $p$-harmonic function in the annular domain $\Omega(r, v)=E(r, v) \backslash D(r, v)$ whose continuous extension to the closure satisfies $u_{r, v}(\partial D(r, v))=1, u_{r, v}(\partial E(r, v))=-1$. Then $\operatorname{dist}\left(0,\left\{u_{r, v}(x)=\alpha_{0}\right\}\right)=r \eta$, where $\eta=\operatorname{dist}\left(0,\left\{u_{1, v}(x)=\alpha_{0}\right\}\right)>0$, since $u_{r, v}(x)=u_{1, v}(x / r)$. For $r=\min \left\{1, \operatorname{dist}\left(\partial K_{1}, \partial K_{3}\right)\right\}$ and any point $x_{0} \in \partial K_{1}$, we have $x_{0}+D\left(r,\left(x_{0} /\left|x_{0}\right|\right)\right) \subset \bar{K}_{1}$ and $x_{0}+E\left(r,\left(x_{0} /\left|x_{0}\right|\right)\right) \subset K_{3}$. By the comparison principle, we have $u(x) \geqslant u_{r, v}\left(x-x_{0}\right)$ in $\Omega \cap\left(x_{0}+\Omega_{r, v}\right)$, where $v=x_{0} /\left|x_{0}\right|$. It follows that $B_{r \eta}\left(x_{0}\right) \subset K_{1} \cup\left\{u(x)<\alpha_{0}\right\}$ for all $x_{0} \in K_{1}$, from which the assertion follows.

\section{The multi-layer case}

Let us recall the problem. We are given two strictly nested convex domains $K_{1} \subset K_{m+2}$, real numbers $-1 \leqslant \lambda_{i} \leqslant 1(i=1,2, \ldots, m+1)$ with $\lambda_{i}>\lambda_{i+1}$, and continuous functions $g_{i}$ : $\left(K_{m+2} \backslash K_{1}\right) \times \mathbb{R}_{+} \rightarrow \mathbb{R}_{+}(i=2, \ldots, m+1)$. We are looking for a sequence of nested convex domains

$$
K_{1} \subset \subset K_{2} \subset \subset \cdots \subset \subset K_{m+1} \subset \subset K_{m+2}
$$

such that the $p$-capacitary potentials $u_{i}(x)$ of the sets $K_{i+1} \backslash \bar{K}_{i}$, i.e. solutions of

$$
\begin{cases}\Delta_{p} u_{i}=0 & \text { in } K_{i+1} \backslash \bar{K}_{i}, \\ u_{i}=\lambda_{i} & \text { on } \partial K_{i}, \\ u_{i}=\lambda_{i+1} & \text { on } \partial K_{i+1},\end{cases}
$$

satisfy the following joining conditions:

$$
\left|\nabla u_{i}(x)\right|=g_{i}\left(x,\left|\nabla u_{i+1}(x)\right|\right) \quad \text { on } \partial K_{i+1} \quad(i=1, \ldots, m) .
$$

For simplicity we set $\lambda_{1}=1$ and $\lambda_{m+1}=-1$. The following is our main result in this paper.

THEOREM 6.1 (multi-layer) Let $K_{1}, K_{m+2}$ be two bounded convex domains such that $K_{m+2}$ strictly contains $K_{1}, \lambda_{i} \in(-1,1), i=2, \ldots, m+1$, are arbitrary real numbers with $\lambda_{i}>\lambda_{i+1}$, and $g_{i} \in \mathcal{G}, i=1, \ldots, m$. Then there exists a sequence $\left\{K_{i}: 1<i<m+2\right\}$ of convex $C^{1}$ domains such that $K_{1} \subset \subset K_{2} \subset \subset \cdots \subset \subset K_{m+1} \subset \subset K_{m+2}$, and which is a classical solution of the multi-layer free boundary problem. The latter means that the $p$-capacitary potentials $u_{i}$ of the sets $K_{i+1} \backslash \bar{K}_{i}, i=1, \ldots, m$, i.e. solutions of 44 satisfy

$$
\lim _{\substack{z \rightarrow x \\ z \in K_{i+1} \backslash \bar{K}_{i}}}\left|\nabla u_{i}(z)\right|=\lim _{\substack{y \rightarrow x \\ y \in K_{i+2} \backslash \bar{K}_{i+1}}} g\left(y,\left|\nabla u_{i+1}(y)\right|\right) \quad \forall x \in \partial K_{i+1}, \quad i=1, \ldots, m .
$$

Definitions We let $\mathcal{B}$ denote the family of all ordered $(m-1)$-tuples $\omega:=\left(\omega_{2}, \omega_{3}, \ldots, \omega_{m+1}\right)$ such that

$$
K_{1} \subset \subset \omega_{2} \subset \subset \cdots \subset \subset \omega_{m+1} \subset \subset K_{m+2},
$$


every $\omega_{i}$ is a convex domain and each domain $\omega_{i}$ is a supersolution of the two-layer problem relative to $\omega_{i-1}$ and $\omega_{i+1}$. The latter means that for every $i=1, \ldots, m$, we have

$$
\underset{\substack{z \rightarrow x \\ z \in \omega_{i+1} \backslash \bar{\omega}_{i}}}{\limsup }\left|\nabla u_{i}(z)\right| \leqslant \liminf _{\substack{y \rightarrow x \\ y \in \omega_{i+2} \backslash \bar{\omega}_{i+1}}} g_{i+1}\left(y,\left|\nabla u_{i+1}(y)\right|\right) \quad \forall x \in \partial \omega_{i+1},
$$

where we take $\omega_{1}:=K_{1}, \omega_{m+2}:=K_{m+2}$ and define $u_{i}$ to be the solution of (44) with $K_{i}$ replaced by $\omega_{i}$ for each $i=1, \ldots, m+1$.

Step 1: Modified two-layer existence result. In view of Theorem 5.1, the proof of Theorem 4.1 actually yields the following result for the two-layer problem: (a) There exists a unique absolute minimizer among all exterior solutions. (b) This absolute minimizer solves the two-layer problem (in the same sense as in Theorem 4.1).

Step 2: $\mathcal{B}$ is not empty. Under our assumptions, an outer $(m-1)$-surface outer solution can be easily obtained in the form $\omega_{i}=\left\{u(x)>\alpha_{i}\right\}, i=2, \ldots, m+1$, where $u$ denotes the solution of (26) with $K_{3}$ replaced by $K_{m+2}$, and where the values $\alpha_{i}$ are appropriately chosen so that each $\omega_{i}$ is a supersolution relative to its neighbors $\omega_{i-1}$ and $\omega_{i+1}$ (same argument as in the first step in the proof of Theorem 4.1p.

Step 3: Uniform separation in $\mathcal{B}$. Let $\omega:=\left(\omega_{2}, \ldots, \omega_{m+1}\right) \in \mathcal{B}$. Then for each $i=2, \ldots, m+1$, $\omega_{i}$ is a supersolution of the two-layer problem relative to $\omega_{i-1}, \omega_{i+1}$, and the function $g_{i}$. Thus, by Theorem[5.1, we have

$$
\operatorname{dist}\left(\partial \omega_{i-1}, \partial \omega_{i}\right) \geqslant \eta \operatorname{dist}\left(\partial \omega_{i-1}, \partial \omega_{i+1}\right) \geqslant \eta \operatorname{dist}\left(\partial \omega_{i}, \partial \omega_{i+1}\right)
$$

for all $i=2, \ldots, m+1$. It follows that

$$
\operatorname{dist}\left(\partial \omega_{i-1}, \partial \omega_{i}\right) \geqslant \eta^{m+2-i} \operatorname{dist}\left(\partial \omega_{m+1}, \partial K_{m+2}\right) \geqslant \eta^{m} \operatorname{dist}\left(\partial \omega_{m+1}, \partial K_{m+2}\right)
$$

for all $i=2, \ldots, m+1$. Thus, if $\omega^{n}, n=1,2, \ldots$, is a weakly decreasing sequence of elements of $\mathcal{B}$ (so that the corresponding sequence of $(m-1)$-st components is also weakly decreasing and thus uniformly bounded away from $\partial K_{m+2}$ ), then there exists a value $\delta>0$ such that for all $n=1,2, \ldots$, the surface components of $\omega^{n}$ are separated from each other (and from $\partial K_{1}$ and $\partial K_{m+2}$ ) by a distance of at least $\delta$. Therefore the componentwise intersection has the same property.

Step 4: Pairwise intersection; minimal sequence in $\mathcal{B}$. $\mathcal{B}$ is closed under the operation of componentwise intersection. In fact, given $\omega^{1}, \omega^{2} \in \mathcal{B}$, let $\omega=\omega^{1} \cap \omega^{2}$ be the componentwise intersection. Then $\partial \omega \subset \partial \omega^{1} \cup \partial \omega^{2}$, and it is easy to see (using the standard comparison principle) that $u^{\omega} \leqslant u^{\omega^{j}}, j=1,2$, componentwise in the common domains of the component $p$-capacitary potentials. By repeated application of componentwise intersections, one defines a (componentwise) weakly decreasing minimal sequence of supersolutions $\omega^{n}=\left(\omega_{2}^{n}, \ldots, \omega_{m+1}^{n}\right), n=1,2, \ldots$, where the latter means that for any $i=2, \ldots, m-1$ and any $x \in \mathbb{R}^{N}$ such that $x \notin \omega_{i}$ for some supersolution $\omega \in \mathcal{B}$, we have $x \notin \omega_{i}^{n}$ for all sufficiently large $n$.

Step 5: Minimal element in $\mathcal{B}$. For each fixed $i=2, \ldots, m+1$, the sequence of domains $\omega_{i}^{n}, n=$ $1,2, \ldots$, is weakly decreasing under set inclusion and therefore convergent to a domain $\Omega_{i} \supset K_{1}$ $\left(\Omega_{i}:=\right.$ the interior of the infinite intersection of the domains $\left.\omega_{i}^{n}, n=1,2, \ldots\right)$. Clearly the domains $\Omega_{i}$ are strictly ordered by inclusion, and in fact by step 3 , we have $\operatorname{dist}\left(\partial \Omega_{i}, \partial \Omega_{i+1}\right) \geqslant \delta$ for all 
$i=1, \ldots, m+1$ (where we set $\Omega_{1}:=K_{1}$ and $\Omega_{m+2}:=K_{m+2}$ ). Since $\Omega_{i} \subset \omega_{i}^{n}$ for all $i$, each $\omega_{i}^{n}, i=2, \ldots, m+1$, is actually a supersolution of the two-layer problem relative to $\Omega_{i-1}, \Omega_{i+1}$, and $g_{i}$. Therefore, $\Omega_{i}$ (the interior of the infinite intersection of the $\omega_{i}^{n}$ ) is also a supersolution of the same two-layer problem, due to Theorem 4.3 Therefore $\Omega \in \mathcal{B}$. In fact $\Omega$ is, by construction, the minimal supersolution in $\mathcal{B}$.

Step 6: $\Omega$ solves the multi-layer problem. Since $\Omega$ is a minimal element in $\mathcal{B}$, each component $\Omega_{i}$ of $\Omega$ must be the minimal supersolution of the two-layer problem relative $\Omega_{i-1}, \Omega_{i+1}$, and $g_{i}$. Therefore, by step $1, \Omega_{i}$ is a solution of this two-layer problem in the sense of Theorem 4.1 Thus Theorem 6.1 is proved.

\section{Acknowledgements}

The authors want to thank the referees whose suggestions improved significantly the writing of this paper.

\section{REFERENCES}

1. ACKER, A. On the multi-layer fluid problem: regularity, uniqueness, convexity and successive approximation of solutions. Comm. Partial Differential Equation 16 (1991), 647-666. Zbl 0732.35109 MR 92k:35290

2. ACKER, A. On the existence of convex classical solutions for multi-layer free boundary problems with general nonlinear joining conditions. Trans. Amer. Math. Soc. 350 (1998), 2981-3020. Zbl 0898.35115 MR 98k:35209

3. ACKER, A. On the non-convexity of solutions in free-boundary problems arising in plasma physics and fluid dynamics. Comm. Pure Appl. Math. 42 (1989), 1165-1174. Zbl 0711.76113 MR 91a:35167 (Addendum: Comm. Pure Appl. Math. 44 (1991), 869-872. Zbl 0753.76198 MR 92h:35243)

4. ACKER, A. Qualitative properties of the boundary derivative of the capacity potential for special classes of annular domains. Math. Methods Appl. Sci. 7 (1985), 251-259. Zbl 0585.35018 MR 86k:31001

5. ACKER, A. On 2-layer free boundary problems with generalized joining conditions: convexity and successive approximation of solutions. Comparison Methods and Stability Theory, X. Liu and D. Siegel (eds.), Lecture Notes in Pure and Appl. Math. 162, Dekker (1994), 1-15. Zbl 0868.35046 MR 95e:35229

6. Alt, H. W. \& CAffarelli, L. A. Existence and regularity for a minimum problem with free boundary. J. Reine Angew. Math. 325 (1981), 105-144. Zbl 0449.35105 MR 83a:49011

7. Dobrowolski, M. On quasilinear elliptic equations in domains with conical boundary points. J. Reine Angew. Math. 394 (1989), 186-195. Zbl 0655.35022 MR 90c:35087

8. Henrot, A. \& Shahgholian, H. Convexity of free boundaries with Bernoulli type boundary condition. Nonlinear Anal. 28 (1997), 815-823. Zbl 0868.35046 MR 97j:35165

9. Henrot, A. \& Shahgholian, H. Existence of classical solutions to a free boundary problem for the $p$ Laplace operator: (I) the exterior convex case. J. Reine Angew. Math. 521 (2000), 85-97. Zbl 0955.35078 MR 2001f:35442

10. Henrot, A. \& Shahgholian, H., Existence of classical solutions to a free boundary problem for the p-Laplace operator: (II) the interior convex case. Indiana Univ. Math. J. 49 (2000), 311-323. Zbl 0977.35148 MR 2001m:35326

11. Henrot, A. \& Shahgholian, H., The one-phase free boundary problem for the $p$-Laplacian with nonconstant Bernoulli boundary condition. Trans. Amer. Math. Soc. 354 (2002), 2399-2416. Zbl 0988.35174 MR 2003c:35165 
12. KROL, I. N. The solutions of the equation $D_{x_{i}}\left(D u^{p-2} D_{x_{i}} u\right)=0$ with a singularity at a boundary point. Boundary Value Problems of Mathematical Physics 8, Tr. Mat. Inst. Steklova 125 (1973), 127-139. MR $49 \# 9409$

13. Laurence, P. \& Stredulinsky, E. Existence of regular solutions with convex levels for semilinear elliptic equations with nonmonotone $L^{1}$ nonlinearities. Part (I). Indiana Univ. Math. J. 39 (1990), 10811114. Zbl 0798.35167 MR 92m:35279

14. Lewis, J. L. Capacitary functions in convex rings. Arch. Rat. Mech. Anal. 66 (1977), 201-224. Zbl 0393.46028 MR 57 \#16638

15. Payne, L. E. \& Philippin, G. On the conformal capacity problem. Geometry of Solutions to Partial Differential Equations, Symposia Math., G. Talenti (ed.), Academic Press, New York (1989), 119-136. Zbl 0714.35017 MR 91h:35067

16. Payne, L. E. \& Philippin, G., On gradient maximum principles for quasilinear elliptic equations, Nonlinear Anal. 23 (1994), 387-398. Zbl 0820.35018 MR 95h:35035

17. Tolksdorf, P. On the Dirichlet problem for quasilinear equations in domains with conical boundary points. Comm. Partial Differential Equations 8 (1983), 773-817. Zbl 0515.35024 MR 85g:35053 